\title{
Research Article \\ Existence of Infinitely Many Distinct Solutions to the Quasirelativistic Hartree-Fock Equations
}

\author{
M. Enstedt ${ }^{1}$ and M. Melgaard ${ }^{2}$ \\ ${ }^{1}$ Department of Mathematics, Uppsala University, 75106 Uppsala, Sweden \\ ${ }^{2}$ School of Mathematical Sciences, Dublin Institute of Technology, Dublin 8, Ireland \\ Correspondence should be addressed to M. Melgaard, michael.melgaard@dit.ie
}

Received 18 March 2009; Revised 8 July 2009; Accepted 10 August 2009

Recommended by Irena Lasiecka

We establish existence of infinitely many distinct solutions to the semilinear elliptic Hartree-Fock equations for $N$-electron Coulomb systems with quasirelativistic kinetic energy $\sqrt{-\alpha^{-2} \Delta_{x_{n}}+\alpha^{-4}}-$ $\alpha^{-2}$ for the $n$th electron. Moreover, we prove existence of a ground state. The results are valid under the hypotheses that the total charge $Z_{\text {tot }}$ of $K$ nuclei is greater than $N-1$ and that $Z_{\text {tot }}$ is smaller than a critical charge $Z_{c}$. The proofs are based on a new application of the Fang-Ghoussoub critical point approach to multiple solutions on a noncompact Riemannian manifold, in combination with density operator techniques.

Copyright (c) 2009 M. Enstedt and M. Melgaard. This is an open access article distributed under the Creative Commons Attribution License, which permits unrestricted use, distribution, and reproduction in any medium, provided the original work is properly cited.

\section{Introduction}

In the present paper we prove existence of infinitely many solutions to the quasirelativistic Hartree-Fock equations

$$
\widetilde{T}_{0} \phi_{n}+V_{\mathrm{en}} \phi_{n}+\left(\rho * \frac{1}{|x|}\right) \phi_{n}-K^{\mathrm{xc}} \phi_{n}+\lambda_{n} \phi_{n}=0, \quad n=1, \ldots, N,
$$

associated with $N$ electrons interacting with $K$ static nuclei with charges $Z_{1}, \ldots, Z_{K}$, where $Z_{k}>0$. The nonlinear coupled equations arise as the Euler-Lagrange equations of the total energy functional $\mathcal{\varepsilon}(\cdot)$ defined as the quantum energy restricted to (antisymmetric) Slater determinants (see Section 3) constructed from $L^{2}$-orthonormal functions $\left\{\phi_{n}\right\}_{n=1}^{N}$ belonging to the Sobolev space $\mathbf{H}^{1 / 2}\left(\mathbb{R}^{3}\right)$. Above $\widetilde{T}_{0}=\sqrt{-\alpha^{-2} \Delta_{x_{n}}+\alpha^{-4}}-\alpha^{-2}$ is the quasirelativistic kinetic energy of the $n$th electron located at $x_{n} \in \mathbb{R}^{3}\left(\Delta_{x_{n}}\right.$ being the Laplacian with respect to $\left.x_{n}\right), \alpha$ is Sommerfeld's fine structure constant, $V_{\mathrm{en}}$ is the attractive interaction between an 
electron and the nuclei, $\rho=\sum_{n=1}^{N}\left|\phi_{n}\right|^{2}$ is the density, and $K^{\mathrm{xc}}$ is the exchange operator defined in (4.1) below. For the nonrelativistic setting, a review on classical results on existence of a ground state and its properties is found in Lions [1]. In the latter paper, Lions studied both minimal and nonminimal (excited states) solutions to the equations by using critical point theory in conjunction with Morse data. Lions' idea is to construct convenient min-max levels which yield the desired solutions through abstract critical point theory. For the nonrelativistic HF model, Lions verifies a Palais-Smale (compactness) condition which, roughly speaking, amounts to "being away from the continuous spectrum" or, equivalently (when the so-called Morse information is taken into account), showing that certain Schrödinger operators with Coulomb type potentials have enough negative eigenvalues.

The novelty of the present paper is Theorem 7.1, wherein we establish the following results for the quasirelativistic Hartree-Fock equations. (1) A ground state exists provided the total charge $Z_{\text {tot }}$ of $K$ nuclei is greater than $N-1$ and $Z_{\text {tot }}$ is smaller than a critical charge $Z_{c}$ to be defined below; (2) under the same assumptions, infinitely many distinct solutions to the quasirelativistic Hartree-Fock equations exist; we refer to the theorem for the full statement. We proceed to sketch the proof of Theorem 7.1, starting with the existence of a ground state. We consider the $C^{2}$-functional $\varepsilon$ on a (Hilbert) manifold $\mathcal{C}_{N}$ defined in (3.7). Since $\varepsilon$ is bounded from below, we may try to find a critical point at the level $l=\inf _{\mathcal{C}_{N}} \varepsilon$ by determining whether the infimum is achieved. As we will see, it is easy to find an almost critical sequence at the level $l$, that is, a sequence $\left\{\mathbf{h}^{(j)}\right\}$ in $\mathcal{C}_{N}$ satisfying

$$
\lim _{j \rightarrow \infty} \mathcal{\varepsilon}\left(\mathbf{h}^{(j)}\right)=l, \quad \lim _{j \rightarrow \infty} \mathcal{E}^{\prime}\left(\mathbf{h}^{(j)}\right)=0 .
$$

The hard part is to prove existence of a converging subsequence of $\left\{\mathbf{h}^{(j)}\right\}$. Unfortunately, roughly speaking due to ionization, the energy functional will not satisfy a Palais-Smale condition at level $l$. To make sure that we can extract a convergent subsequence, we use second-order information of $\varepsilon$.

In the process of implementing these ideas we have to overcome additional technicalities for the quasirelativistic setting compared to the nonrelativistic, for instance, the Coulomb potential is not relatively compact (in the operator sense) with respect to the quasirelativistic energy operator. In particular, compact Sobolev imbeddings are not available (for a recent survey of such problems, we refer to Bartsch et al. [2]). To overcome this problem, it is necessary to switch to a density operator formalism, as pioneered by Solovej [3], and use that for an enlarged set of admissible density operators, one can, at least for the certain sequences, establish the inequality (6.20) below. A different proof for existence of a ground state was given by Dall'Acqua et al. [4]. Moreover, regularity of the ground state away from the nucleus and pointwise exponential decay of the orbitals were established therein.

In the opposite direction, Lieb [5] has proved that for $N \geq 2 Z_{\text {tot }}+K$ there never exists a quasirelativistic Hartree-Fock ground state (see Enstedt and Melgaard [6] for an analogous result). For the nonrelativistic setting, Solovej has improved Lieb's result by proving that there exists a universal constant $Q>0$ such that $N \geq Z+Q$ ensures that there are no minimizers [7] and Lewin [8] has applied Lions' approach to the nonrelativistic MCSCF equations. For further references, we refer to the survey by Le Bris and Lions $[9$, Section 3.1.6].

We invoke a direct method developed by Fang and Ghoussoub $[10,11]$ to address the existence of infinitely many nonminimal solutions. Since we are looking for nonminimal (or 
unstable) critical points, we consider a collection $\mathrm{H}$ of compact subsets of $\mathcal{C}_{N}$ which is stable under a specific class of homotopies and then we show that $\mathcal{\varepsilon}$ has a critical point at the level

$$
l=l_{\varepsilon, \mathrm{H}}=\inf _{\mathrm{M} \in \mathrm{H}} \max _{\mathrm{h} \in \mathrm{M}} \mathcal{\varepsilon}(\mathbf{h})
$$

As we will see, the method by Fang and Ghoussoub gives us an almost critical sequence at the level $l$, that is, a sequence $\left\{\mathbf{h}^{(j)}\right\}$ in $\mathcal{C}_{N}$ satisfying (1.2), with additional Morse information (as mentioned above) which is crucial for proving that the sequence is convergent.

Work related to our study of semilinear elliptic equations and critical point theory includes existence of solutions with finite Morse indices established by Dancer [12], de Figueiredo et al. [13], Flores et al. [14], and Tanaka [15], existence of multiple solutions established by Cingolani and Lazzo [16] and Ghoussoub and Yuan [17], "relaxed" PalaisSmale sequences as in Lazer and Solimini [18] and Jeanjean [19], and problems on noncompact Riemannian manifolds found in Fieseler and Tintarev [20, 21], Mazepa [22], and Tanaka [23].

\section{Preliminaries}

Throughout the paper we denote by $\mathrm{c}$ and $C$ (with or without indices) various positive constants whose precise value is of no importance. Moreover, we will denote the complex conjugate of $z \in \mathbb{C}$ by $\bar{z}$.

\section{Function Spaces}

For $1 \leq p \leq \infty$, let $L^{p}\left(\mathbb{R}^{3}\right)$ be the space of (equivalence classes of) complex-valued functions $\phi$ which are measurable and satisfy $\int_{\mathbb{R}^{3}}|\phi(x)|^{p} d x<\infty$ if $p<\infty$ and $\|\phi\|_{L^{\infty}\left(\mathbb{R}^{3}\right)}=\operatorname{ess} \sup |\phi|<\infty$ if $p=\infty$. The measure $d x$ is the Lebesgue measure. For any $p$ the $L^{p}\left(\mathbb{R}^{3}\right)$ space is a Banach space with norm $\|\cdot\|_{L^{p}\left(\mathbb{R}^{3}\right)}=\left(\left.\int_{\mathbb{R}^{3}} \cdot\right|^{p} d x\right)^{1 / p}$. In the case $p=2, L^{2}\left(\mathbb{R}^{3}\right)$ is a complex and separable Hilbert space with scalar product $\langle\phi, \psi\rangle_{L^{2}\left(\mathbb{R}^{3}\right)}=\int_{\mathbb{R}^{3}} \phi \bar{\psi} d x$ and corresponding norm $\|\phi\|_{L^{2}\left(\mathbb{R}^{3}\right)}=$ $\langle\phi, \phi\rangle_{L^{2}\left(\mathbb{R}^{3}\right)}^{1 / 2}$. Similarly, $L^{2}\left(\mathbb{R}^{3}\right)^{N}$, the $N$-fold Cartesian product of $L^{2}\left(\mathbb{R}^{3}\right)$, is equipped with the scalar product $\langle\phi, \psi\rangle=\sum_{n=1}^{N}\left\langle\phi_{n}, \psi_{n}\right\rangle_{L^{2}\left(\mathbb{R}^{3}\right)}$. The space of infinitely differentiable complexvalued functions with compact support will be denoted $C_{0}^{\infty}\left(\mathbb{R}^{3}\right)$. The Fourier transform is given by

$$
(\mp \psi)(\xi)=\widehat{\psi}(\xi)=(2 \pi)^{-3 / 2} \int_{\mathbb{R}^{3}} e^{-i x \xi} \psi(x) d x
$$

Define

$$
\mathbf{H}^{1 / 2}\left(\mathbb{R}^{3}\right)=\left\{\phi \in L^{2}\left(\mathbb{R}^{3}\right):(1+|\xi|)^{1 / 2} \widehat{\phi} \in L^{2}\left(\mathbb{R}^{3}\right)\right\}
$$

which, equipped with the scalar product

$$
\langle\phi, \psi\rangle_{\mathbf{H}^{1 / 2}\left(\mathbb{R}^{2}\right)}=\int_{\mathbb{R}^{3}}(1+|\xi|) \widehat{\phi}(\xi) \overline{\widehat{\psi}(\xi)} d \xi
$$


becomes a Hilbert space; evidently, $\mathbf{H}^{1}\left(\mathbb{R}^{3}\right) \subset \mathbf{H}^{1 / 2}\left(\mathbb{R}^{3}\right)$. We have that $C_{0}^{\infty}\left(\mathbb{R}^{3}\right)$ is dense in $\mathbf{H}^{1 / 2}\left(\mathbb{R}^{3}\right)$ and the continuous embedding $\mathbf{H}^{1 / 2}\left(\mathbb{R}^{3}\right) \hookrightarrow L^{3}\left(\mathbb{R}^{3}\right)$ holds; more precisely, the Sobolev inequality

$$
\|\phi\|_{L^{3}\left(\mathbb{R}^{3}\right)}^{2} \leq c_{\text {sob }}\|\phi\|_{\mathbf{H}^{1 / 2}\left(\mathbb{R}^{3}\right)}^{2}
$$

is valid with $c_{\text {sob }}=2^{-1 / 2} \pi^{-2 / 3}$. Moreover, we will use any weakly convergent sequence that in $\mathbf{H}^{1 / 2}\left(\mathbb{R}^{3}\right)$ has a pointwise convergent subsequence.

\section{Operators}

Let $T$ be a self-adjoint operator on a Hilbert space $\mathscr{L}$ with domain $\mathfrak{D}(T)$. The spectrum and resolvent set are denoted by $\sigma(T)$ and $\rho(T)$, respectively. We use standard terminology for the various parts of the spectrum; see, for example, [24, 25]. The resolvent is $R(\zeta)=(T-\zeta)^{-1}$. The spectral family associated to $T$ is denoted by $E_{T}(\lambda), \lambda \in \mathbb{R}$. For a lower semibounded self-adjoint operator $T$, the counting function is defined by

$$
\operatorname{Coun}(\lambda ; T)=\operatorname{dim} \operatorname{Ran} E_{T}((-\infty, \lambda))
$$

The space of trace operators, respectively, Hilbert-Schmidt operators, on $\mathfrak{h}=L^{2}\left(\mathbb{R}^{3}\right)$ is denoted by $\mathfrak{S}_{1}(\mathfrak{h})$, respectively, $\mathfrak{S}_{2}(\mathfrak{h})$.

We need the following abstract operator result by Lions [1, Lemma II.2].

Lemma 2.1. Let $T$ be a self-adjoint operator on a Hilbert space $\mathfrak{d e}$, and let $\mathfrak{L}_{1}, \mathfrak{l}_{2}$ be two subspaces of $\mathscr{H}$ such that $\mathscr{H}=\mathscr{H}_{1} \oplus \mathscr{H}_{2}, \operatorname{dim} \mathscr{H}_{1}=\mathrm{h}_{1}<\infty$ and $P_{2} T P_{2} \geq 0$, where $P_{2}$ is the orthogonal projection onto $\mathfrak{L}_{2}$. Then $\mathrm{T}$ has at most $\mathrm{h}_{1}$ negative eigenvalues.

\section{The Quasirelativistic Hartree-Fock Model}

Within the Born-Oppenheimer approximation, the quantum energy of $N$ quasirelativistic electrons interacting with $K$ static nuclei with charges $\mathbf{Z}=\left(Z_{1}, \ldots, Z_{K}\right), Z_{k}>0$, is, in Rydberg units, given by

$$
\begin{aligned}
\mathcal{E}^{\mathrm{QM}}(\cdot) & : \bigwedge_{1}^{N} \mathbf{H}^{1 / 2}\left(\mathbb{R}^{3}, \mathbb{C}\right) \subset L^{2}\left(\mathbb{R}^{3 N}, \mathbb{C}\right) \longrightarrow \mathbb{R}: \Psi_{\mathrm{e}} \\
\mapsto & \alpha^{-1} \sum_{n=1}^{N} \int_{\mathbb{R}^{3 N}}\left|\mathcal{F}_{\mathrm{n}} \Psi_{\mathrm{e}}\left(x_{1}, \ldots, x_{n-1}, \xi, x_{n+1}, \ldots, x_{N}\right)\right|^{2} d x_{1} \cdots d \mu(\xi) \cdots d x_{N} \\
& +\int_{\mathbb{R}^{3 N}}\left(-\alpha^{-2}+V_{\mathrm{en}}\left(x_{n}\right)+\frac{1}{2} \sum_{m \neq n} V_{\mathrm{ee}}\left(x_{m}-x_{n}\right)\right)\left|\Psi_{\mathrm{e}}\left(x_{1}, \ldots, x_{N}\right)\right|^{2} d x_{1} \cdots d x_{N},
\end{aligned}
$$


where $x=\left(x_{1}, \ldots, x_{N}\right) \in \mathbb{R}^{3 N}, x_{n}=\left(x_{n}^{(1)}, x_{n}^{(2)}, x_{n}^{(3)}\right) \in \mathbb{R}^{3}$ is the position of the $n$th electron, $\alpha$ is Sommerfeld's fine structure constant, and the potentials $V_{\text {ee }}$ and $V_{\text {en }}$ are given by

$$
V_{\mathrm{ee}}(x)=\frac{1}{|x|}, \quad V_{\mathrm{en}}(y)=\sum_{k=1}^{K} V_{k}(y), \quad V_{k}(y)=-\frac{Z_{k} \alpha}{\left|y-R_{k}\right|}
$$

with $R_{k} \in \mathbb{R}^{3}$ being the position of the $k$ th nucleus. Here it is important that $Z_{\text {tot }}<Z_{c}:=$ $2 /(\alpha \pi)$. See Section 3.1 for details. In what follows, we ignore the spin variable but the entire contents can be trivially carried over to the spin-valued setting. Above $\mathcal{F}_{\mathrm{n}} \Psi_{\mathrm{e}}$ is the Fourier transform of $\Psi_{\mathrm{e}}\left(x_{1}, \ldots, x_{n-1}, \cdot, x_{n+1}, \ldots, x_{N}\right)$, in the case when $N=1$ we will just write $\mathscr{F} \Psi_{\mathrm{e}}$, and

$$
d \mu(\xi):=\sqrt{|\xi|^{2}+\alpha^{-2}}
$$

The interpretation of the quadratic form (3.1) is as follows (see Section 3.1 for its well definedness). The first term corresponds to the quasirelativistic kinetic energy of the electrons, the second term is the one-particle attractive interaction between the electrons and the nuclei, and the third term is the standard two-particle repulsive interaction between the electrons. The wave function $\Psi_{\mathrm{e}}: \mathbb{R}^{3 N} \rightarrow \mathbb{C}$ in (3.1) belongs to $\mathscr{L}_{\mathrm{e}}=\wedge^{N} \mathbf{H}^{1 / 2}\left(\mathbb{R}^{3}\right)$, that is, the $N$-particle Hilbert space consist of antisymmetric functions (expressing the Pauli exclusion principle)

$$
\Psi_{\mathrm{e}}\left(x_{1}, \ldots, x_{N}\right)=\operatorname{sign}(\sigma) \Psi_{\mathrm{e}}\left(x_{\sigma(1)}, \ldots, x_{\sigma(N)}\right) \quad \text { a.e., } \forall \sigma \in S_{N},
$$

where $S_{N}$ is the group of permutations of $\{1, \ldots, N\}$, with the signature of a permutation $\sigma$ being denoted by sign $(\sigma)$, and $\mathbf{H}^{1 / 2}\left(\mathbb{R}^{3}\right)$ is the Sobolev space introduced in Section 2 . The ground state energy is defined as

$$
E^{\mathrm{QM}}(N, \mathbf{Z})=\inf \left\{\mathcal{E}^{\mathrm{QM}}\left(\Psi_{e}\right): \Psi_{e} \in \mathscr{\ell}_{e},\left\|\Psi_{e}\right\|_{L^{2}\left(\mathbb{R}^{3 N}\right)}=1\right\}
$$

To determine $E^{\mathrm{QM}}(N, \mathbf{Z})$ directly turns out to be too difficult, even for small $N$. One of the classical approximation methods for determining $E^{\mathrm{QM}}(N, \mathbf{Z})$ is the Hartree-Fock theory, introduced by Hartree and improved by Fock and Slater in the late 1920s (see, e.g., [26]), which consists of restricting attention to simple wedge products $\Psi_{\mathrm{e}} \in \mathcal{S}_{N}$, where

$$
\mathcal{S}_{N}=\left\{\Psi_{\mathrm{e}} \in \mathscr{L}_{\mathrm{e}}: \exists \Phi=\left\{\phi_{n}\right\}_{1 \leq n \leq N} \in \mathcal{C}_{N} \text { s.t. } \Psi_{\mathrm{e}}=\frac{1}{\sqrt{N !}} \operatorname{det}\left(\phi_{n}\left(x_{m}\right)\right)\right\}
$$

with

$$
\mathcal{C}_{N}=\left\{\Phi=\left\{\phi_{n}\right\}_{1 \leq n \leq N}, \phi_{n} \in \mathbf{H}^{1 / 2}\left(\mathbb{R}^{3}\right),\left\langle\phi_{m}, \phi_{n}\right\rangle_{L^{2}}=\delta_{m n}, 1 \leq m, n \leq N\right\} .
$$

This space is clearly a complete metric space and also an (Hilbert) manifold. A function $\Psi_{e} \in$ $\mathcal{S}_{N}$ is sometimes called a Slater determinant, and the $\phi_{n}$ are called orbitals [26]. 
In fact, if $\Psi_{\mathrm{e}} \in \mathcal{S}_{N}$ then, by simple algebraic calculations, $\mathcal{E}^{\mathrm{QM}}\left(\Psi_{\mathrm{e}}\right)=\mathcal{E}\left(\Psi_{\mathrm{e}}\right)$, where the quasirelativistic Hartree-Fock functional (or simply the energy functional) $\mathcal{\varepsilon}(\cdot):\left(\mathbf{H}^{1 / 2}\left(\mathbb{R}^{3}\right)^{N} \rightarrow\right.$ $\mathbb{R}$ is given by

$$
\begin{aligned}
\mathcal{E}\left(\Psi_{\mathrm{e}}\right)= & \mathcal{E}\left(\phi_{1}, \ldots, \phi_{N}\right)=\alpha^{-1} \sum_{n=1}^{N} \int_{\mathbb{R}^{3}}\left|\mp \phi_{n}\right|^{2} d \mu(\xi)-\int_{\mathbb{R}^{3}} \alpha^{-1}\left|\phi_{n}\right|^{2} d x \\
& +\alpha^{-1} \int_{\mathbb{R}^{3}} V_{\mathrm{en}}(x) \rho(x) d x+\frac{1}{2} \iint_{\mathbb{R}^{3}} \frac{\rho(x) \rho\left(x^{\prime}\right)-\left|\Phi\left(x, x^{\prime}\right)\right|^{2}}{\left|x-x^{\prime}\right|} d x d x^{\prime}
\end{aligned}
$$

Here

$$
\bigoplus\left(x, x^{\prime}\right)=\sum_{n=1}^{N} \phi_{n}(x) \overline{\phi_{n}\left(x^{\prime}\right)}
$$

is the density matrix, and

$$
\rho_{\Phi}(x)=\sum_{n=1}^{N}\left|\phi_{n}(x)\right|^{2}
$$

is the density associated to the state $\Psi_{\mathrm{e}}$; when there is no risk of confusion we will suppress the dependence of $\boldsymbol{\Phi}$.

By standard arguments (see, e.g., [27, Lemma 4.1]) we obtain the following result on the regularity of quasirelativistic functional $\mathcal{E}(\cdot)$.

Lemma 3.1. The functional $\varepsilon(\cdot)$ belongs to $C^{2}\left(\mathbf{H}^{1 / 2}\left(\mathbb{R}^{3}\right)^{N}, \mathbb{R}\right)$.

Analogous to (3.5) we define what follows

Definition 3.2 (quasirelativistic Hartree-Fock ground state). Let $\mathbf{Z}=\left(Z_{1}, \ldots, Z_{K}\right), Z_{k}>0$, $k=1, \ldots, K$, and let $N$ be a nonnegative integer. The quasirelativistic Hartree-Fock ground state energy is

$$
E(N, \mathbf{Z})=\inf \left\{\mathcal{E}\left(\Psi_{\mathrm{e}}\right): \Psi_{\mathrm{e}} \in \mathcal{S}_{N}\right\}
$$

and if it is attained we say that the molecule has a quasirelativistic Hartree-Fock ground state described by $\Psi_{\mathrm{e}}$.

\subsection{Atomic and Molecular Hamiltonians}

By $p$ we denote the momentum operator $-i \nabla$ on $L^{2}\left(\mathbb{R}^{3}\right)$. The operator $T_{0}=\sqrt{p^{2}+\alpha^{-2}}$ is generated by the closed positive form $\mathfrak{t}_{0}[\phi, \phi]=\left\langle T_{0}^{1 / 2} \phi, T_{0}^{1 / 2} \phi\right\rangle_{L^{2}\left(\mathbb{R}^{3}\right)}$ on the form domain $\mathfrak{D}\left(\mathfrak{t}_{0}\right)=\mathbf{H}^{1 / 2}\left(\mathbb{R}^{3}\right)$. Set $S(x)=Z \alpha /|x|, Z>0, Z_{\mathrm{c}}=2 \alpha^{-1} \pi^{-1}$, and let $\widetilde{T}_{0}=T_{0}-\alpha^{-1}$. The following facts are well known for the perturbed one-particle operator $H_{1,1, \alpha}=\widetilde{T}_{0}-S(x)[25,28]$. 


\section{Small Perturbations}

If $Z<(\pi / 2) Z_{\mathrm{c}}$ then $S$ is $\widetilde{T}_{0}$-bounded with relative bound equal to two. If, on the other hand, $(2 \alpha)^{-1}<Z<Z_{\mathrm{c}}$ then $S$ is $\widetilde{T}_{0}$-form bounded with relative bound less than one.

We prove the above-mentioned form boundedness. It follows from the following inequality (first observed, it seems, by Kato [25, paragraph V-5.4]):

$$
\langle S \phi, \phi\rangle_{L^{2}\left(\mathbb{R}^{3}\right)} \leq\left(\frac{Z}{Z_{\mathrm{c}}}\right)\|\phi\|_{\mathbf{H}^{1 / 2}\left(\mathbb{R}^{3}\right)^{\prime}}^{2} \quad \forall \phi \in \mathbf{H}^{1 / 2}\left(\mathbb{R}^{3}\right)
$$

Indeed, if, for any $\psi, \phi \in \mathbf{H}^{1 / 2}\left(\mathbb{R}^{3}\right)$, we define the sesquilinear forms

$$
\begin{aligned}
& \mathfrak{s}[\psi, \phi]:=\left\langle S^{1 / 2} \psi, S^{1 / 2} \phi\right\rangle_{L^{2}\left(\mathbb{R}^{3}\right)^{\prime}} \\
& \mathfrak{t}_{0}[\psi, \phi]:=\left\langle T_{0}^{1 / 2} \psi, T_{0}^{1 / 2} \phi\right\rangle_{L^{2}\left(\mathbb{R}^{3}\right)^{\prime}} \\
& \tilde{\mathfrak{t}}_{0}[\psi, \phi]:=\mathfrak{t}_{0}[\psi, \phi]-\alpha^{-1}\langle\psi, \phi\rangle_{L^{2}\left(\mathbb{R}^{3}\right)^{\prime}}
\end{aligned}
$$

then (3.12) shows that $\mathfrak{s}$ is well defined and also, by invoking $|-i \nabla| \leq T_{0}$, we infer that, for all $\phi \in \mathbf{H}^{1 / 2}\left(\mathbb{R}^{3}\right)$,

$$
\mathfrak{s}[\phi, \phi]<\mathfrak{t}_{0}[\phi, \phi] \text { provided } Z<Z_{\mathrm{c}} .
$$

This is the Coulomb uncertainty principle in the quasirelativistic setting. The KLMN theorem (see, e.g., [25, paragraph VI-1.7]) implies that there exists a unique self-adjoint operator, denoted $H_{1,1, \alpha}$, generated by the closed sesquilinear form

$$
\mathfrak{h}_{1,1, \alpha}[\psi, \phi]:=\tilde{\mathfrak{t}}_{0}[\psi, \phi]-\mathfrak{s}[\psi, \phi], \quad \psi, \phi \in \mathfrak{D}\left(\mathfrak{h}_{1,1, \alpha}\right)=\mathbf{H}^{1 / 2}\left(\mathbb{R}^{3}\right)
$$

which is bounded below by $-\alpha^{-1}$. It is well known [28] that

$$
\begin{gathered}
\sigma\left(H_{1,1, \alpha}\right) \cap\left[-\alpha^{-1}, 0\right) \text { is discrete } \\
\sigma\left(H_{1,1, \alpha}\right) \cap[0, \infty) \text { is absolutely continuous. }
\end{gathered}
$$

In particular,

$$
\sigma_{\mathrm{ess}}\left(H_{1,1, \alpha}\right)=[0, \infty) .
$$


The form construction of the atomic Hamiltonian $H_{1,1, \alpha}$ can be generalized to the molecular case, describing a molecule with $N$ electrons and $K$ nuclei of charges $\mathbf{Z}=\left(Z_{1}, \ldots, Z_{K}\right), Z_{k}>$ 0 , located at $R_{1}, \ldots, R_{K}, R_{k} \in \mathbb{R}^{3}$, if we substitute $\mathfrak{s}$ by

$$
\mathfrak{v}_{\mathrm{en}}[\psi, \phi]=\sum_{k=1}^{K}\left\langle V_{k}^{1 / 2}, \psi, V_{k}^{1 / 2} \phi\right\rangle, \quad \psi, \phi \in \mathbf{H}^{1 / 2}\left(\mathbb{R}^{3}\right)
$$

where $V_{k}$ is defined in (3.2) and by assuming that $Z_{\text {tot }}<Z_{c}$. Under the same hypothesis, we note that the discussion on the forms $\mathfrak{s}, \mathfrak{t}_{0}$, and $\mathfrak{v}_{\text {en }}$ immediately gives us that the form (3.1) (and thus $\varepsilon(\cdot)$ ) is well defined closed and bounded from below.

\subsection{Density Operator Formalism}

We can re-express $\mathcal{\varepsilon}(\cdot)$ and the Hartree-Fock ground state energy via the one-to-one correpondence between elements of $\mathcal{C}_{N}$ and projections onto finite-dimensional subspaces of $L^{2}\left(\mathbb{R}^{3}\right)$. Indeed, given an element $\left\{\phi_{n}\right\}_{n=1}^{N}$ in $\mathcal{C}_{N}$ we can associate a canonical projection operator, $\boldsymbol{\Phi}=\sum_{n=1}^{N}\left\langle\cdot, \phi_{n}\right\rangle \phi_{n}$ with trace equal to $N$. We may therefore write

$$
\mathcal{E}(\boldsymbol{\Phi})=\alpha^{-1}\left(\operatorname{Tr}\left[\widetilde{T}_{0} \boldsymbol{\Phi}\right]-\operatorname{Tr}\left[V_{\mathrm{en}} \boldsymbol{\Phi}\right]\right)+2(\boldsymbol{\Phi})-\mathcal{K}(\boldsymbol{\Phi})
$$

where

$$
\begin{aligned}
\operatorname{Tr}\left[\widetilde{T}_{0} \oslash\right] & =\sum_{n=1}^{N} \mathfrak{t}_{0}\left[\phi_{n}, \phi_{n}\right]-\alpha^{-1}\left[\phi_{n}, \phi_{n}\right], \\
\operatorname{Tr}\left[V_{\mathrm{en}} \oslash\right] & =\sum_{n=1}^{N} \mathfrak{v}_{\mathrm{en}}\left[\phi_{n}, \phi_{n}\right] .
\end{aligned}
$$

The direct Coulomb energy defined in terms of the Coulomb inner product

$$
\partial(\boldsymbol{\Phi})=2\left(\rho_{\Phi}, \rho_{\Phi}\right)=\frac{1}{2} \iint_{\mathbb{R}^{3}} \rho_{\Phi}(x)\left|x-x^{\prime}\right|^{-1} \rho_{\Phi}\left(x^{\prime}\right) d x d x^{\prime}
$$

and the exchange Coulomb energy defined by

$$
\mathcal{K}(\Phi):=\frac{1}{2} \iint_{\mathbb{R}^{3}} \frac{\left|\Phi\left(x, x^{\prime}\right)\right|^{2}}{\left|x-x^{\prime}\right|} d x d x^{\prime}
$$

Furthermore, it is not hard to verify that given a projection operator with trace $N$ defined on $L^{2}\left(\mathbb{R}^{3}\right)$ we can also find an element in $\mathcal{C}_{N}$ corresponding to this operator. It is therefore clear that the Hartree-Fock ground state energy can be expressed as

$$
E(N, \mathbf{Z})=\inf \left\{\mathcal{\varepsilon}(\boldsymbol{\Phi}): \boldsymbol{\Phi}^{*}=\boldsymbol{\Phi}=\boldsymbol{\Phi}^{2}, \operatorname{Tr}[\boldsymbol{\Phi}]=N\right\}
$$


More generally, a density operator $\Phi$ is a trace class operator on $\mathfrak{h}=L^{2}\left(\mathbb{R}^{3}\right)$, in symbols $\Phi \in \mathfrak{S}_{1}(\mathfrak{h})$, which satisfies the operator inequality $0 \leq \mathscr{\Phi} \leq I$. This motivates the following definition:

$$
\mathcal{M}_{\mathrm{adm}}=\left\{\boldsymbol{\Phi} \in \mathfrak{S}_{1}(\mathfrak{h}): 0 \leq \boldsymbol{\Phi} \leq I, \operatorname{Tr}[\boldsymbol{\Phi}]=N\right\}
$$

Using standard arguments [3] in combination with (3.12), and the Sobolev inequality (2.4), it is easy to show that $\mathcal{\varepsilon}(\cdot)$ is well defined on the following enlarged set of density operators:

$$
\mathcal{M}_{\mathrm{enl}}=\left\{\boldsymbol{\Phi} \in \mathcal{M}_{\mathrm{adm}}: \operatorname{Tr}\left[T_{0} \boldsymbol{\Phi}\right]<+\infty\right\}
$$

For later purpose we also introduce

$$
\mathcal{M}_{\text {proj }}=\left\{\Phi \in \mathcal{M}_{\mathrm{enl}}: \Phi^{2} \geq \Phi\right\}
$$

\section{The Quasirelativistic Fock Operator}

Herein we introduce the quasirelativistic Fock operator.

Lemma 4.1. Assume $Z_{\text {tot }}<Z_{c}$. Let

$$
K^{\mathrm{xc}}\left(x, x^{\prime}\right)=\frac{\bigoplus\left(x, x^{\prime}\right)}{\left|x-x^{\prime}\right|}
$$

be the integral kernel of the exchange operator $K^{\mathrm{xc}}$. Then the unique self-adjoint operator $F$ associated with the differential expression

$$
\alpha^{-1} \widetilde{T}_{0} \phi+\alpha^{-1} V_{\mathrm{en}} \phi+\rho * \frac{1}{|x|} \phi-K^{\mathrm{xc}} \phi
$$

is generated by the sesquilinear form

$$
\begin{aligned}
\mathfrak{f}[\phi, \psi]= & \alpha^{-1} \widetilde{\mathfrak{t}}_{0}[\phi, \psi]+\alpha^{-1} \int_{\mathbb{R}^{3}} V_{\text {en }}(x) \phi(x) \bar{\psi}(x) d x \\
& +\iint_{\mathbb{R}^{3}} \frac{\rho(x) \phi(y) \bar{\psi}(y)}{|x-y|} d x d y-\iint_{\mathbb{R}^{3}} \Phi(x, y) \frac{\phi(y) \bar{\psi}(x)}{|x-y|} d y d x .
\end{aligned}
$$

Proof. Bear in mind the definitions of $\mathfrak{t}_{0}, \widetilde{\mathfrak{t}}_{0}$, and $\mathfrak{v}_{\text {en }}$ from Section 3.1. Define $\mathfrak{v}_{\rho *(1 /|x|)}$ as the third form on the right-hand side of (4.3). Then (3.12) yields the estimate

$$
\mathfrak{v}_{\rho *(1 /|x|)}[\phi, \phi] \leq C\left(Z_{\mathrm{c}}, N\right) \mathfrak{t}_{0}[\phi, \phi] \text {. }
$$

Under the hypothesis, we already know from Section 3.1 that the quadratic form $\mathfrak{t}_{0}+\mathfrak{v}_{\text {en }}$ is nonnegative on $\mathbf{H}^{1 / 2}\left(\mathbb{R}^{3}\right)$. Evidently, $\mathfrak{v}_{\rho *(1 /|x|)}$ is a nonnegative form and, consequently, 
$\tilde{\mathfrak{f}}=\mathfrak{t}_{0}+\mathfrak{v}_{\mathrm{en}}+\mathfrak{v}_{\rho *(1 /|x|)}$ is a nonnegative form on $\mathbf{H}^{1 / 2}\left(\mathbb{R}^{3}\right)$. Closedness of the nonnegative quadratic form $\tilde{\mathfrak{f}}$ is equivalent to lower semicontinuity of $\tilde{f}$ on $\mathbf{H}^{1 / 2}\left(\mathbb{R}^{3}\right)$. In fact, $\tilde{\mathfrak{f}}$ is continuous. Indeed, (3.12), respectively, (4.4) enables us to show continuity of the second, respectively, the third terms, in $\tilde{\mathfrak{f}}$. For instance, we consider $\mathfrak{v}_{\text {en }}$ and assume that $\phi_{j} \rightarrow \phi$ in $\mathbf{H}^{1 / 2}\left(\mathbb{R}^{3}\right)$. Then an application of Hölder's inequality and (3.12) yields

$$
\begin{aligned}
& \left|\mathfrak{v}_{\text {en }}\left[\phi_{j}\right]-\mathfrak{v}_{\text {en }}[\phi]\right| \leq \int_{\mathbb{R}^{3}}\left|V_{\text {en }}\right|\left|\phi_{j}-\phi\right|\left|\phi_{j}+\phi\right| d x \\
& \quad \leq \mathrm{c}\left(\int_{\mathbb{R}^{3}}\left|V_{\text {en }}\right|\left|\phi_{j}-\phi\right|^{2} d x\right)^{1 / 2} \leq C\left\|\phi_{j}-\phi\right\|_{\mathbf{H}^{1 / 2}\left(\mathbb{R}^{3}\right)} .
\end{aligned}
$$

We conclude that $\tilde{f}$ is a closed quadratic form on $\mathbf{H}^{1 / 2}\left(\mathbb{R}^{3}\right)$. The first representation theorem [24, Theorem VI.2.4] informs us that the nonnegative closed form $\tilde{f}$ is associated to a unique self-adjoint operator, say $\widetilde{F}$. Furthermore, the exchange operator $K^{\mathrm{xc}}$ is a Hilbert-Schmidt operator. Indeed, using, in this particular order, the weak Young inequality, the Hölder inequality and (3.12) we find that $K^{\mathrm{xc}}\left(x, x^{\prime}\right) \in L^{2}\left(\mathbb{R}^{3} \times \mathbb{R}^{3}\right)$. It is clear that the form $\tilde{\mathrm{f}}[\cdot, \cdot \cdot]-$ $\alpha^{-1}\langle\cdot, \cdot\rangle_{L^{2}}-\left\langle K^{\mathrm{xc}} \cdot, \cdot\right\rangle_{L^{2}}$ is closed and, once again applying the first representation theorem, we obtain a unique self-adjoint operator $F$ associated with the form in (4.3).

\section{Lower Spectral Bound}

We will later need the following spectral result.

Lemma 5.1. Assume $\vartheta<Z_{\text {tot }}<Z_{c}$, and let $\rho \in L^{1}\left(\mathbb{R}^{3}\right) \cap L^{4 / 3}\left(\mathbb{R}^{3}\right)$ such that $\int_{\mathbb{R}^{3}} \rho d x<\vartheta$. Define the quasirelativistic Schrödinger operator

$$
T=\alpha^{-1} \widetilde{T}_{0}+\alpha^{-1} V_{\text {en }}+\rho * \frac{1}{|x|}
$$

Then, for any $\kappa \geq 1$ and any $0 \leq \vartheta<Z_{\mathrm{tot}}$, there exists $\epsilon_{\kappa, \vartheta}>0$ such that

$$
\text { Count }\left(-\epsilon_{n, \vartheta} ; T\right) \geq \kappa
$$

Proof. By a minor modification of [28, page 291], which carries over the result (3.17) from the one-nucleus to the many-nuclei cases, we deduce that the essential spectrum of $\widetilde{T}_{0}+V_{\text {en }}$ equals the semiaxis $[0, \infty)$. Next, a standard perturbation argument and (yet) an application of Weyl's essential spectrum theorem prove that $\sigma_{\text {ess }}(T)=[0, \infty)$. Let $\mathfrak{t}_{\mu}$ denote the quadratic form defined by

$$
\alpha^{-1} \widetilde{\mathfrak{t}}_{0}[\phi, \phi]+\int_{\mathbb{R}^{3}}\left(\alpha^{-1} V_{\text {en }}+\rho * \frac{1}{|x|}\right)|\phi(x)|^{2} d x .
$$


For any $\kappa \geq 1$ and any $0 \leq \vartheta \leq Z_{\text {tot }}$ we construct a $\kappa$-dimensional subspace $\mathscr{H}_{\kappa, \vartheta}$ in $\mathbf{H}^{1 / 2}\left(\mathbb{R}^{3}\right)$ such that

$$
\mathfrak{t}[\phi, \phi]<-\epsilon_{\kappa, \vartheta}<0
$$

for all $L^{2}$-normalized $\phi \in \mathscr{H}_{\kappa, \vartheta}$. We note that

$$
\alpha^{-1} \widetilde{\mathfrak{t}}_{0}[\phi, \phi] \leq C \int_{\mathbb{R}^{3}}|\nabla \phi|^{2} d x
$$

As a consequence, by selecting a $\mathcal{\kappa}$-dimensional subspace of normalized radially symmetric functions in $C_{0}^{\infty}\left(\mathbb{R}^{3}\right)$, we can construct a subspace $\mathscr{H}_{\kappa, \vartheta}$ of functions satisfying (5.4), away from $\phi=0$, by repeating the arguments in [1, Lemma II.1] (see also [27, Lemma 6.1]). Then the assertion follows by an application of Glazman's Lemma.

Within the nonrelativistic context a similar result was first given by Lions [1, Lemma II.1].

\section{Relative Compactness of Palais-Smale Type Sequences}

In this section we give the main auxiliary result that will be used in the proof of Theorem 7.1. We emphasize that the functional $\varepsilon(\cdot)$ is not weakly lower semicontinuous on $\left(\mathbf{H}^{1 / 2}\left(\mathbb{R}^{3}\right)\right)^{N}$ and, in the proof below, it is thus necessary to switch to a density operator formalism. In particular, we use that for a specific sequence of density operators (see the proof for details), one can establish the inequality (6.20) below (replacing the notion of weak lower semicontinuity which is absent).

Proposition 6.1. Assume that $l \in \mathbb{R}$, that $m \in \mathbb{N}$, and let $N-1<Z_{\mathrm{tot}}<Z_{c}$. Then any sequence $\left\{\phi^{(j)}\right\}_{j=1}^{\infty} \subset \mathcal{C}_{N}$ satisfying a Palais-Smale condition at level $l$ and of order less than $m$ is relatively compact in $\mathcal{C}_{N}$, that is, any sequence $\left\{\phi^{(j)}\right\}_{j=1}^{\infty}$ in $\mathcal{C}_{N}$ is relatively compact whenever the sequence satisfies the following conditions:

(i) $\lim _{j \rightarrow \infty} \varepsilon\left(\phi^{(j)}\right)=l$;

(ii) $\lim _{j \rightarrow \infty} \varepsilon^{\prime}\left(\phi^{(j)}\right)=0$;

(iii) there exists a sequence of positive reals $\left\{\delta^{(j)}\right\}_{j=1}^{\infty}$ with $\delta^{(j)} \rightarrow 0$ such that for each $j, \varepsilon^{\prime \prime}\left(\phi^{(j)}\right)$ has at most $m$ eigenvalues below $-\delta^{(j)}$.

Moreover, the components of the limit element $\phi=\left(\phi_{1}, \ldots, \phi_{N}\right)$ of $\left\{\phi^{(j)}\right\}_{j=1}^{\infty}$ in $\mathcal{C}_{N}$ satisfy the quasirelativistic Hartree-Fock equations

$$
\begin{gathered}
F \phi_{n}+\lambda_{n} \phi_{n}=0, \quad n=1, \ldots, N, \\
\left\langle\phi_{m}, \phi_{n}\right\rangle_{L^{2}\left(\mathbb{R}^{3}\right)}=\delta_{m n}
\end{gathered}
$$

where $\lambda_{n} \geq 0$ for $Z_{\text {tot }}>N-1$, respectively, $\lambda_{n}>0$ for $Z_{\text {tot }}>N$, and $F$ is the Fock operator defined in Lemma 4.1. 
Proof. First we treat the case $N<Z_{\text {tot. }}$. Henceforth we let $\left\{\phi_{n}^{(j)}\right\}_{n=1}^{N} \in \mathcal{C}_{N}$ be the canonical sequence associated with an operator $\Phi^{(j)}$ in $\mathcal{M}_{\text {proj }}$ defined in Section 3.2. The hypotheses (i) and (ii) give us that

$$
\sup _{\|\psi\|_{\mathbf{H}^{1 / 2\left(\mathbb{R}^{3}\right)}}=1}\left|\mathfrak{f}_{j}\left[\phi_{n}^{(j)}, \psi\right]+\lambda_{n}^{(j)}\left\langle\phi_{n}^{(j)}, \psi\right\rangle\right| \longrightarrow 0, \quad \forall n,
$$

where $\lambda_{n}^{(j)}$ is a sequence of reals and $\mathfrak{f}_{j}$ is a sequence of quadratic forms associated with $\left\{\phi_{n}^{(j)}\right\}$, defined as in (4.3).

Let us now extract some subsequences that we will need. Let us start by proving existence of $0<\lambda<\tilde{\lambda}<\infty$ such that $\lambda<\lambda_{n}^{(j)}<\tilde{\lambda}$. To prove existence of a lower bound we note that from hypothesis (iii) we get (in particular) that

$$
\mathfrak{f}_{j}[\psi, \psi]+\left(\lambda_{n}^{(j)}+\delta_{n}^{(j)}\right)\|\psi\|_{L^{2}\left(\mathbb{R}^{3}\right)}^{2} \geq 0
$$

with $\delta_{n}^{(j)} \rightarrow 0$ in the standard Euclidean metric for each fixed $n$ and $\psi$ in a closed subspace of $\mathbf{H}^{1 / 2}\left(\mathbb{R}^{3}\right)$ with finite codimension $N+m$. By invoking Lemma 2.1 we deduce that the quasirelativistic Schrödinger operator

$$
T^{(j)}=\widetilde{T}_{0}+V_{\text {en }}+\rho^{(j)} * \frac{1}{|x-y|}
$$

has at most $N+m$ eigenvalues strictly less than $-\left(\lambda_{n}^{(j)}+\delta_{n}^{(j)}\right)$. Moreover, since

$$
\int_{\mathbb{R}^{3}} \rho^{(j)} d x=\sum_{n=1}^{N} \int_{\mathbb{R}^{3}}\left|\phi_{n}^{(j)}(x)\right|^{2}=N<Z_{\text {tot }}
$$

Lemma 5.1 ensures that there exists $\delta>0$ (independent of $j$ ) such that $T^{(j)}$ has at least $N+m$ eigenvalues strictly below $-\delta$. As a consequence, we infer that

$$
\lambda_{n}^{(j)}+\delta_{n}^{(j)} \geq \delta, \quad \forall j
$$

Since $\delta_{n}^{(j)} \rightarrow 0$ as $j \rightarrow \infty$, we conclude that, for $j$ large enough,

$$
\lambda_{n}^{(j)} \geq \lambda>0, \quad \forall n
$$

We note that the hypothesis $Z_{\text {tot }}<Z_{c}$ and the fact that $\left\{\boldsymbol{\Phi}^{(j)}\right\} \subset \mathcal{M}_{\text {proj }}$ satisfies

$$
\varepsilon\left(\Phi^{(j)}\right) \longrightarrow l
$$


ensure the existence of a constant $C$, depending on $N$, such that

$$
\operatorname{Tr}\left[T_{0} \Phi^{(j)}\right] \leq C\left(1+\operatorname{Tr}\left[\Phi^{(j)}\right]\right) .
$$

To prove existence of an upper bound we note that

$$
0 \leq \sum_{n=1}^{N} \lambda_{n}^{(j)} \leq-\varepsilon\left(\Phi^{(j)}\right) \leq \widetilde{C}
$$

which follows from the Cauchy-Schwarz inequality and (6.8). Now, perhaps after going to a subsequence using the Bolzano-Weierstrass theorem, we may assume that

$$
\lambda_{n}^{(j)} \longrightarrow \lambda_{n}>0
$$

We know from (6.9) that

$$
\operatorname{Tr}\left[T_{0} \Phi^{(j)}\right]
$$

is uniformly bounded in $j$. Then some straightforward calculations give us that

$$
\operatorname{Tr}\left[T_{0}^{1 / 2} \boldsymbol{\Phi}^{(j)} T_{0}^{1 / 2}\right]
$$

is also uniformly bounded in $j$. Here the $T_{0}^{1 / 2}$ is defined using Kato's second representation theorem [24, Theorem VI.2.4] . Hence we may, using the Banach-Alaoglu theorem, extract a subsequence such that $T_{0}^{1 / 2} \boldsymbol{\Phi}^{(j)} T_{0}^{1 / 2}$ converges weakly in $\mathfrak{S}_{2}$ to an element $\tilde{\boldsymbol{\Phi}}$. Fix any $\psi \in$ $L^{2}\left(\mathbb{R}^{3}\right)$, then

$$
\langle\cdot \psi, \psi\rangle: \mathfrak{S}_{1} \longrightarrow \mathbb{R}
$$

is a linear bounded functional. We get that

$$
\lim _{j}\left\langle\boldsymbol{\Phi}^{(j)} \psi, \psi\right\rangle=\lim _{j}\left\langle T_{0}^{1 / 2} \boldsymbol{\Phi}^{(j)} T_{0}^{1 / 2} T_{0}^{-1 / 2} \psi, T_{0}^{-1 / 2} \psi\right\rangle=\left\langle T_{0}^{-1 / 2} \tilde{\Phi} T_{0}^{-1 / 2} \psi, \psi\right\rangle .
$$

Define $\Phi:=T_{0}^{-1 / 2} \tilde{\Phi} T_{0}^{-1 / 2}$ and let $\left\{\psi_{n}\right\}_{n=1}^{\infty} \subset \mathbf{H}^{1 / 2}\left(\mathbb{R}^{3}\right)$ be a basis in $L^{2}\left(\mathbb{R}^{3}\right)$. Then a direct application of Fatou's lemma (with respect to a counting measure) gives us that

$$
\operatorname{Tr}[\Phi] \leq \lim _{j} \inf \operatorname{Tr}\left[\Phi^{(j)}\right] \leq N
$$

Mutatis mutandis it is clear that

$$
\operatorname{Tr}\left[T_{0} \Phi\right] \leq \lim _{j} \inf \operatorname{Tr}\left[T_{0} \Phi^{(j)}\right] \leq C
$$


We note that $\Phi^{(j)}$ converges weakly to $\Phi$ in $\mathfrak{S}_{2}$ and hence that the kernels of the operators $\Phi^{(j)}$ will converge weakly in $L^{2}\left(\mathbb{R}^{3} \times \mathbb{R}^{3}\right)$ to the kernel of $\boldsymbol{\Phi}$. In view of (6.17) and the fact that $\operatorname{Tr}\left[\Phi^{(j)}\right] \leq C$ we infer that there exists a subsequence such that

$$
\phi_{n}^{(j)} \longrightarrow \phi_{n} \in \mathbf{H}^{1 / 2}\left(\mathbb{R}^{3}\right)
$$

weakly in $\mathbf{H}^{1 / 2}\left(\mathbb{R}^{3}\right)$ and, by invoking weak compactness (see Section 2), the convergence holds almost everywhere. Since weak limits are unique, we may assume that the kernel associated with $\Phi$ can be written as

$$
\Phi\left(x, x^{\prime}\right):=\sum_{n=1}^{N} \phi_{n}(x) \overline{\phi_{n}\left(x^{\prime}\right)}
$$

The inequality

$$
\lim _{j} \inf \mathcal{\varepsilon}\left(\boldsymbol{\Phi}^{(j)}\right) \geq \mathcal{E}(\boldsymbol{\Phi})
$$

can be derived by arguments similar to the ones in [4, pages 722-724], wherein it is proven for a minimizing sequence (bearing in mind the spectral properties of the one-particle operator $H_{1,1, \alpha}$ in (3.15) which we summarized in Section 3.1). More specically, using arguments by Barbaroux et al. [26] and Solovej [30], the inequality (6.20) was proved by Dall'Acqua et al [16] (for specfic sequences) in the quasirelativistic setting and their proof carries over to our sequence. As a consequence, we have that

$$
\begin{aligned}
\limsup _{j} \sum_{n=1}^{N} \lambda_{n}^{(j)}\left\|\phi_{n}{ }^{(j)}\right\|_{L^{2}}^{2} & =-\lim _{j} \inf \mathcal{E}\left(\boldsymbol{\Phi}^{(j)}\right)+\frac{1}{2}\left(\partial\left(\boldsymbol{\Phi}^{(j)}\right)-\mathcal{K}\left(\boldsymbol{\Phi}^{(j)}\right)\right) \\
& \leq-\mathcal{E}(\boldsymbol{\Phi})-\frac{1}{2}(\partial(\boldsymbol{\Phi})-\mathcal{\mathcal { K }}(\boldsymbol{\Phi}))=\sum_{n=1}^{N} \lambda_{n}\left\|\phi_{n}\right\|_{L^{2}}^{2} \\
& \leq \lim _{j} \inf \sum_{n=1}^{N} \lambda_{n}^{(j)}\left\|\phi_{n}{ }^{(j)}\right\|_{L^{2}}^{2} .
\end{aligned}
$$

From this we conclude that $\operatorname{Tr}[\Phi]=N$ and therefore that $\left\|\phi_{n}\right\|_{L^{2}\left(\mathbb{R}^{3}\right)}=1$. Repeating the argument above, we obtain the convergence in $\mathbf{H}^{1 / 2}\left(\mathbb{R}^{3}\right)^{N}$. We recall the regularity property of $\mathcal{E}$ and that the quasirelativistic Hartree-Fock equations are the Euler-Lagrange equations corresponding to this functional. The last assertion then follows from hypothesis (ii) and the relative compactness that was just proved. inequality

Finally, we consider the case $N-1<Z_{\text {tot }}$. By going to the limit in (6.3), the resulting

$$
\mathfrak{f}[\psi, \psi]+\lambda_{n}\|\psi\|_{L^{2}\left(\mathbb{R}^{3}\right)}^{2} \geq 0
$$


holds on a closed subspace of $\mathbf{H}^{1 / 2}\left(\mathbb{R}^{3}\right)$ with finite codimension; this requires that $\phi_{n}^{(j)} \rightarrow \phi_{n}$ weakly in $\mathbf{H}^{1 / 2}\left(\mathbb{R}^{3}\right)$ (inspection of the argument above justifies this). Hence we infer that $T$ (defined similar to $T^{(j)}$ with $\rho^{(j)}$ replaced by $\rho$ ) has at most finitely many eigenvalues less than or equal to $-\lambda_{n} \leq 0$. If $\operatorname{Tr}[\nexists]=N$, then we are done. If, on the other hand, $\operatorname{Tr}[\nexists]<N$ then we apply Lemma 5.1 and repeat the reasoning above. This completes the proof. [3].

The density operator argument in the proof of Proposition 6.1 is inspired by Solovej

Remark 6.2. It is worth to mention that from the perspective of Physics, there is no difference between the requirements $Z_{\text {tot }}>N-1$ and $Z_{\text {tot }} \geq N$ because $Z_{\text {tot }}$ is integer valued.

\section{Existence of a Ground State and Excited States}

The main result is the following theorem.

Theorem 7.1. Assume that the total nuclear charge $Z_{\mathrm{tot}}=\sum_{k=1}^{K} Z_{k}$ satisfies $Z_{\mathrm{tot}}<Z_{c}$ and let $N \in \mathbb{N}$ satisfy $N-1<Z_{\text {tot }}$. Then

(1) every minimizing sequence of the quasirelativistic Hartree-Fock functional $\varepsilon(\cdot)$ is relatively compact in $\mathcal{C}_{N}$. In particular, there exists a minimizer $\varphi$ of $\mathcal{E}(\cdot)$ on the admissible set $\mathcal{C}_{N}$ and (up to unitary transformations) the components of $\varphi=\left(\varphi_{1}, \ldots, \varphi_{N}\right)$ satisfy the quasirelativistic HartreeFock equations

$$
\begin{gathered}
F \varphi_{n}+\lambda_{n} \varphi_{n}=0, \\
\left\langle\varphi_{m}, \varphi_{n}\right\rangle_{L^{2}\left(\mathbb{R}^{3}\right)}=\delta_{m n}
\end{gathered}
$$

where $F$ is the quasirelativistic Fock operator defined in Lemma 4.1, and the numbers $-\lambda_{n}$ are the $N$ lowest negative eigenvalues of $F$,

(2) there exists a sequence $\left\{\varphi^{(k)}\right\}_{k \geq 1}$, with entries $\varphi^{(k)}=\left(\varphi_{1}^{(k)}, \ldots, \varphi_{N}^{(k)}\right)$, of distinct solutions of the quasirelativistic Hartree-Fock equations (7.1) in $\mathbf{H}^{1 / 2}\left(\mathbb{R}^{3}\right)^{N}$ which satisfy the constraints $\left\langle\varphi_{m}^{(k)}, \varphi_{n}^{(k)}\right\rangle_{L^{2}\left(\mathbb{R}^{3}\right)}=\delta_{m n}$ for all $1 \leq m, n \leq N$ and, furthermore, the Lagrange multipliers $\lambda_{n}^{(k)}$ are positive, respectively, nonnegative, when $Z_{\text {tot }}>N$, respectively, $Z_{\text {tot }}>N-1$. Moreover, the following properties are valid as $k \rightarrow \infty$ :

$$
\begin{gathered}
\lambda_{n}^{(k)} \longrightarrow 0, \quad \varepsilon\left(\varphi^{(k)}\right) \longrightarrow 0, \\
\varphi^{(k)} \longrightarrow 0 \quad \text { weakly in } \mathbf{H}^{1 / 2}\left(\mathbb{R}^{3}\right)^{N},
\end{gathered}
$$

(3) any solution to (7.1) belongs to $\mathbb{C}^{\infty}\left(\mathbb{R}^{3} \backslash\left\{R_{1}, \ldots, R_{N}\right\}\right)^{N}$ and $\varphi_{n}$ decays exponentially sufficiently far away from the locations of the nuclei.

Before proving assertion 1 of Theorem 7.1, let us give a few explanations. To ensure that a Palais-Smale sequence converges, one needs to somehow "improve" it. Since $\varepsilon$ is a $C^{2}$ functional, one may try to obtain an almost critical sequence with some information on the second derivative. This enables us to built an almost critical sequence which satisfies (6.3). 
Due to lack of compactness, one cannot find critical points of $\mathcal{E}$ and therefore one perturbs the functional while, simultaneous, ensuring that the new functional has critical points of the kind, one expects for the original one. The way one will obtain such sequences consist in applying a "perturbed variational principle" by Borwein and Preiss [30].

Proof of Theorem 7.1 (assertion 1). First of all we note that using (3.12) and the Cauchy-Schwarz inequality that $\mathcal{E}$ is bounded from below uniformly on $\mathcal{C}_{N}$ and we may therefore conclude existence of a minimizing sequence, $\left\{\widetilde{\Phi}^{(j)}\right\}_{j=1}^{\infty}$ to (3.11). To prove relative compactness we will now prove that the hypotheses (ii) and (iii) in Proposition 6.1 are satisfied. An application of the Borwein and Preiss variational principle [30, Theorem 2.6] provides us with a new minimization sequence $\left\{\Phi^{(j)}\right\}_{j=1}^{\infty}$, such that

$$
\left\|\Phi^{(j)}-\widetilde{\Phi}^{(j)}\right\|_{\mathbf{H}^{1 / 2}\left(\mathbb{R}^{3}\right)^{N}} \longrightarrow 0
$$

We will also have that $\Phi^{(j)}$ minimizes

$$
\mathcal{\varepsilon}(\cdot)+\gamma^{(j)}\left\|\cdot-\Psi^{(j)}\right\|_{\mathbf{H}^{1 / 2}\left(\mathbb{R}^{3}\right)^{N}}^{2}
$$

for some $\Psi^{(j)} \in \mathbf{H}^{1 / 2}\left(\mathbb{R}^{3}\right)^{N}$ and $\gamma^{(j)}>0$, where $\gamma^{(j)} \rightarrow 0$. From this we can conclude that hypothesis (ii) is satisfied. If we then follow the idea to prove a lower bound on the reals in the proof of Proposition 6.1 it is not difficult to show that hypothesis (iii) is satisfied for $N$. Existence of a minimum follows from Proposition 6.1. To show that $\lambda_{n}>0$ one argues by contradiction as in [27, page 2139]. The last assertion on the Lagrange multipliers and its relation to the Fock operator has been proven in [4].

Proceeding towards the second assertion of Theorem 7.1 which addresses the existence of infinitely many nonminimal solutions, one would expect from the previous proof that a more involved perturbed variational principle is needed. For our specific setting, however, it suffices to apply the direct method by Fang and Ghoussoub [10] (see also [11, 31]). Again, due to the lack of weakly lower semicontinuity, it is necessary to switch to density operators in the proof below.

Proof of Theorem 7.1 (assertions 2 and 3). We will prove that there exists a critical point at infinitely many distinct levels. We will use abstract critical point theory by Fang and Ghoussoub [10]. Consider the $C^{2}$-functional $\varepsilon$ on the $C^{2}$-Riemannian manifold $\mathcal{C}_{N}$. We consider $\mathbb{Z}_{2}=\{0,1\}$ to be the compact (0-dimensional) Lie group, with groups actions $(0, \phi) \mapsto \phi$ and $(1, \phi) \mapsto-\phi \quad\left(\phi \in \mathcal{C}_{N}\right)$. We note that the functional is even, in fact it is invariant under unitary transformation, this can be seen by repeating the proof from the nonrelativistic Hartree-Fock case (see, e.g., [27, Lemma 2.3]. Next we make preparations for the min-max principle: For each $k \in \mathbb{N}$, we consider the following homotopic classes of order $k$

$$
\mathrm{H}_{k}=\left\{\mathrm{M}: \mathrm{M}=h\left(\mathbb{S}^{k-1}\right) \text { for some odd continuous } h: \mathbb{S}^{k-1} \rightarrow \mathcal{C}_{N}\right\},
$$


where $\mathbb{S}^{k-1}$ is the unit sphere in the Euclidean space $\mathbb{R}^{k}$. Let

$$
l_{k}=\inf _{M \in H_{k}} \max _{\phi \in \mathbb{M}} \varepsilon(\phi) .
$$

We claim that $-\infty<l_{k} \leq l_{k+1}<0$ for each $k \in \mathbb{N}$ and that $\lim _{k \rightarrow \infty} l_{k}=0$, the proof of this fact will be given last in this proof. We may of course, after perhaps going to a subsequence, assume that $l_{k}<l_{k+1}$ for each $k$. Now, we will use the abstract results by Fang and Ghoussoub [10] (in particular [31, Theorem 11.1 and Remark 11.13]), to extract a sequence satisfying the assumptions of Palais-Smale condition at level $l_{k}$ and of order less than $k$, but such a sequence is according to Proposition 6.1 relatively compact in $\mathcal{C}_{N}$.

Let us now prove the properties of the sequence $\left\{\varphi^{(k)}\right\}_{k \geq 1}$ of distinct solutions. We have already seen that we may assume that $-\infty<l_{k}<l_{k+1}<0$ so we may find a sequence such that

$$
-\infty<l_{k-1}<l_{k}=\mathcal{E}\left(\varphi^{(k)}\right)<l_{k+1}<0 .
$$

We conclude that

$$
\varepsilon\left(\varphi^{(k)}\right) \longrightarrow 0
$$

Now using the Cauchy-Schwarz inequality (recall that $0<\lambda_{n}^{(k)}$ )

$$
-\sum_{n=1}^{N} \lambda_{n}^{(k)} \leq \varepsilon\left(\varphi^{(k)}\right)<0
$$

and thus $\lambda_{n}^{(k)} \rightarrow 0$ in $k$ for each $n$. We note that the right-hand side of

$$
2\left(\varepsilon\left(\varphi^{(k)}\right)+\sum_{n=1}^{N} \lambda_{n}^{(k)}\right)=-\int_{\mathbb{R}^{6}} \frac{\rho^{(k)}(x) \rho^{(k)}\left(x^{\prime}\right)-\left|\Phi^{(k)}\left(x, x^{\prime}\right)\right|^{2}}{\left|x-x^{\prime}\right|} d x d x^{\prime}
$$

tends to zero. This together with (7.7) allows us to conclude that we can find (perhaps after going to a subsequence) a weak limit, $\varphi \in \mathbf{H}^{1 / 2}\left(\mathbb{R}^{3}\right)^{N}$, for our sequence. Due to the assumption $Z_{\mathrm{tot}}<Z_{\mathrm{c}}$ we may find a constant $C>0$ such that

$$
\varepsilon\left(\varphi^{(k)}\right)-\iint_{\mathbb{R}^{3}} \frac{\rho^{(k)}(x) \rho^{(k)}\left(x^{\prime}\right)-\left|\Phi^{(k)}\left(x, x^{\prime}\right)\right|^{2}}{\left|x-x^{\prime}\right|} d x d x^{\prime} \leq C \mathfrak{t}_{0}[\varphi, \varphi]
$$


and we may therefore conclude that $\varphi=0$. This finishes the part on the properties of the sequence. It remains to prove the claim stated above. The monotonicity of $\left\{l_{k}\right\}_{k=1}^{\infty}$ is a direct consequence of how we have defined $\mathrm{H}_{k}$ and since $\mathcal{\varepsilon}$ is uniformly bounded below on $\mathcal{C}_{N}$, we immediately get that $l_{k}>-\infty$. An application of Lemma 5.1 ensures that there exists a $k$ dimensional subspace $\mathscr{L}_{k}$ of $\mathbf{H}^{1 / 2}\left(\mathbb{R}^{3}\right)$ such that for all $\phi \in \mathscr{L}_{k}$ with $\|\phi\|_{L^{2}\left(\mathbb{R}^{3}\right)}=1$ (we denote the unit sphere in this subspace by $\left.\widetilde{\mathbb{S}}^{k-1}\right)$, one has

$$
\tilde{\mathfrak{t}}_{0}[\phi, \phi]+\mathfrak{v}_{e}[\phi, \phi]+\iint_{\mathbb{R}^{3}} \frac{\rho(x)\left|\phi\left(x^{\prime}\right)\right|^{2}}{\left|x-x^{\prime}\right|} d x d x^{\prime} \leq-\epsilon_{k}
$$

for some $\epsilon_{k}>0$. It is not hard to find a continuous isomorphism $g: \mathscr{l}_{k} \rightarrow \mathbb{R}^{k}$ such that $g\left(\mathbb{S}^{k-1}\right)=\widetilde{\mathbb{S}}^{k-1}$, now denote by $e$ the natural embedding of $\widetilde{\mathbb{S}}^{k-1}$ into $\mathcal{C}_{N}$ (due to the monotonicity we may assume $k$ to be sufficiently large) and therefore $g \circ e$ will be an odd and continuous mapping from $\mathbb{S}^{k-1}$ into $\mathcal{C}_{N}$ where $\mathcal{\varepsilon}$ negative and therefore we can conclude that $l_{k}<0$. Hence we can find $\mathrm{M}_{k} \in \mathrm{H}_{k}$ such that

$$
l_{k} \leq \max _{M_{k}} \varepsilon<\frac{l_{k}}{2}
$$

To prove that $\lim _{k \rightarrow \infty} l_{k}=0$, we use the separability of $\mathbf{H}^{1 / 2}\left(\mathbb{R}^{3}\right)$ by considering a nested sequence of finite-dimensional subspaces $\mathcal{W}_{k}$ of $\mathbf{H}^{1 / 2}\left(\mathbb{R}^{3}\right)$ such that $\operatorname{dim}\left(\mathcal{W}_{k}\right)=k$ and $\cup_{k} \mathcal{W}_{k}$ is dense in $\mathbf{H}^{1 / 2}\left(\mathbb{R}^{3}\right)$. Define $\mho_{k}$ as the orthogonal complement of $\mathcal{W}_{k-1}$. Now, assume that $\mathrm{M}_{k} \cap \mathcal{V}_{k}=\emptyset$, let $\pi_{k-1}$ be the orthogonal projection from $\mathbf{H}^{1 / 2}\left(\mathbb{R}^{3}\right)$ onto $\mathfrak{W}_{k-1}$. Then $\left(\mathcal{V}_{k}:=\right.$ $\left.\operatorname{ker}\left(\pi_{k-1}\right)\right)$

$$
\pi\left(\mathrm{M}_{k}\right) \subset \mathcal{W}_{k} \backslash\{0\} \cong \mathbb{R}^{k} \backslash\{0\},
$$

and by following Rabinowitz [32] and using the Borsuk-Ulam theorem we will now arrive at a contradiction. Using that zero is an upper bound for the functional we can extract a sequence $h_{k} \in \mathrm{M}_{k} \cap \mho_{k}$ such that $h_{k}$ tends weakly to some element that must be equal to 0 . By repeating the arguments in Proposition 6.1 we may find a subsequence (which is of course sufficient in our case) such that $\boldsymbol{\Phi}^{(k)}$, the density operator corresponding to $h_{k}$, tends weakly to 0 in $\mathfrak{S}_{2}$. We get by the same type of argument as for (6.20) that

$$
0=\varepsilon(0) \leq \lim \inf _{k} \varepsilon\left(\Phi^{(k)}\right) \leq 0
$$

The latter together with (7.13) implies that $\lim _{k \rightarrow \infty}, l_{k}=0$.

The regularity and decay properties of our sequence were proved in [4] for an atom and it carries over to our setting mutatis mutandis.

\section{Acknowledgment}

The research of the second author is supported by a Stokes Award (Science Foundation Ireland). 


\section{References}

[1] P.-L. Lions, "Solutions of Hartree-Fock equations for Coulomb systems," Communications in Mathematical Physics, vol. 109, no. 1, pp. 33-97, 1987.

[2] T. Bartsch, Z.-Q. Wang, and M. Willem, "The Dirichlet problem for superlinear elliptic equations," in Stationary Partial Differential Equations. Vol. II, Handbook of Differential Equations, pp. 1-55, Elsevier/North-Holland, Amsterdam, The Netherlands, 2005.

[3] J. P. Solovej, "Proof of the ionization conjecture in a reduced Hartree-Fock model," Inventiones Mathematicae, vol. 104, no. 2, pp. 291-311, 1991.

[4] A. Dall'Acqua, T. Ø. Sørensen, and E. Stockmeyer, "Hartree-Fock theory for pseudorelativistic atoms," Annales Henri Poincaré, vol. 9, no. 4, pp. 711-742, 2008.

[5] E. H. Lieb, "Bound on the maximum negative ionization of atoms and molecules," Phys. Rev. A, vol. 29, pp. 3018-3028, 1984.

[6] M. Enstedt and M. Melgaard, "Non-existence of a minimizer to the magnetic Hartree-Fock functional," Positivity, vol. 12, pp. 653-666, 2008.

[7] J. P. Solovej, "The ionization conjecture in Hartree-Fock theory," Ann. of Math (2), vol. 158, no. 2, pp. 509-576, 2003.

[8] M. Lewin, "Solutions to the multiconfiguration equations in quantum chemistry," Arch Rat. Mech. Anal., vol. 171, pp. 83-114, 2004.

[9] C. Le Bris and P.-L. Lions, "From atoms to crystals: a mathematical journey," Bull. Amer. Math. Soc. (N.S.), vol. 42, no. 3, pp. 291-363, 2005.

[10] G. Fang and N. Ghoussoub, "Second-order information on Palais-Smale sequences in the mountain pass theorem," Manuscripta Mathematica, vol. 75, no. 1, pp. 81-95, 1992.

[11] G. Fang and N. Ghoussoub, "Morse-type information on Palais-Smale sequences obtained by minmax principles," Communications on Pure and Applied Mathematics, vol. 47, no. 12, pp. 1595-1653, 1994.

[12] E. N. Dancer, "Finite Morse index solutions of exponential problems," Annales de l'Institut Henri Poincaré. Analyse Non Linéaire, vol. 25, no. 1, pp. 173-179, 2008.

[13] D. G. de Figueiredo, P. N. Srikanth, and S. Santra, "Non-radially symmetric solutions for a superlinear Ambrosetti-Prodi type problem in a ball," Communications in Contemporary Mathematics, vol. 7, no. 6, pp. 849-866, 2005.

[14] G. Flores, P. Padilla, and Y. Tonegawa, "Higher energy solutions in the theory of phase transitions: a variational approach," Journal of Differential Equations, vol. 169, no. 1, pp. 190-207, 2001.

[15] K. Tanaka, "Morse indices at critical points related to the symmetric mountain pass theorem and applications," Communications in Partial Differential Equations, vol. 14, no. 1, pp. 99-128, 1989.

[16] S. Cingolani and M. Lazzo, "Multiple semiclassical standing waves for a class of nonlinear Schrödinger equations," Topological Methods in Nonlinear Analysis, vol. 10, no. 1, pp. 1-13, 1997.

[17] N. Ghoussoub and C. Yuan, "Multiple solutions for quasi-linear PDEs involving the critical Sobolev and Hardy exponents," Transactions of the American Mathematical Society, vol. 352, no. 12, pp. 57035743, 2000.

[18] A. C. Lazer and S. Solimini, "Nontrivial solutions of operator equations and Morse indices of critical points of min-max type," Nonlinear Analysis: Theory, Methods E Applications, vol. 12, no. 8, pp. 761-775, 1988.

[19] L. Jeanjean, “On the existence of bounded Palais-Smale sequences and application to a LandesmanLazer-type problem set on $\mathbb{R}^{N}$," Proceedings of the Royal Society of Edinburgh. Section A, vol. 129, no. 4, pp. 787-809, 1999.

[20] K.-H. Fieseler and K. Tintarev, "Semilinear elliptic problems and concentration compactness on noncompact Riemannian manifolds," The Journal of Geometric Analysis, vol. 13, no. 1, pp. 67-75, 2003.

[21] K. Tintarev and K.-H. Fieseler, Concentration Compactness: Functional-Analytic Grounds and Applications, Imperial College Press, London, UK, 2007.

[22] E. A. Mazepa, "On the existence of entire solutions of a semilinear elliptic equation on noncompact Riemannian manifolds," Matematicheskie Zametki, vol. 81, no. 1, pp. 153-156, 2007 (Russian), translation in: Mathematical Notes, vol. 81, no. 1-2, pp. 135-139, 2007.

[23] K. Tanaka, "Periodic solutions for singular Hamiltonian systems and closed geodesics on noncompact Riemannian manifolds," Annales de l'Institut Henri Poincaré. Analyse Non Linéaire, vol. 17, no. 1, pp. 1-33, 2000.

[24] D. E. Edmunds and W. D. Evans, Spectral Theory and Differential Operators, Oxford Mathematical Monographs, Clarendon Press, Oxford University Press, New York, NY, USA, 1987. 
[25] T. Kato, Perturbation Theory for Linear Operators, Classics in Mathematics, Springer, Berlin, Germany, 1995.

[26] A. Szabo and N. S. Ostlund, Modern Quantum Chemistry: An Introduction to Advanced Electronic Structure Theory, MacMillan, New York, NY, USA, 1982.

[27] M. Enstedt and M. Melgaard, "Existence of a solution to Hartree-Fock equations with decreasing magnetic fields," Nonlinear Analysis: Theory, Methods \& Applications, vol. 69, no. 7, pp. 2125-2141, 2008.

[28] I. W. Herbst, "Spectral theory of the operator $\left(p^{2}+m^{2}\right)^{1 / 2}-Z e^{2} / r$," Communications in Mathematical Physics, vol. 53, no. 3, pp. 285-294, 1977.

[29] J.-M. Barbaroux, W. Farkas, B. Helffer, and H. Siedentop, "On the Hartree-Fock equations of the electron-positron field," Communications in Mathematical Physics, vol. 255, no. 1, pp. 131-159, 2005.

[30] J. M. Borwein and D. Preiss, "A smooth variational principle with applications to subdifferentiability and to differentiability of convex functions," Transactions of the American Mathematical Society, vol. 303, no. 2, pp. 517-527, 1987.

[31] N. Ghoussoub, Duality and Perturbation Methods in Critical Point Theory, vol. 107 of Cambridge Tracts in Mathematics, Cambridge University Press, Cambridge, UK, 1993.

[32] P. H. Rabinowitz, "Variational methods for nonlinear eigenvalue problems," in Eigenvalues of NonLinear Problems (Centro Internaz. Mat. Estivo (C.I.M.E.), III Ciclo, Varenna, 1974), pp. 139-195, Edizioni Cremonese, Rome, Italy, 1974. 


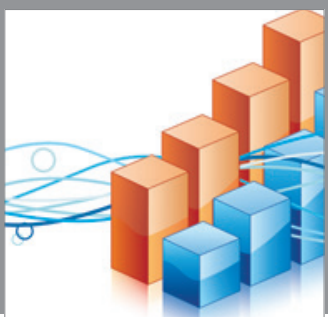

Advances in

Operations Research

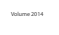

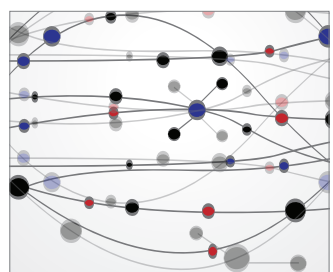

\section{The Scientific} World Journal
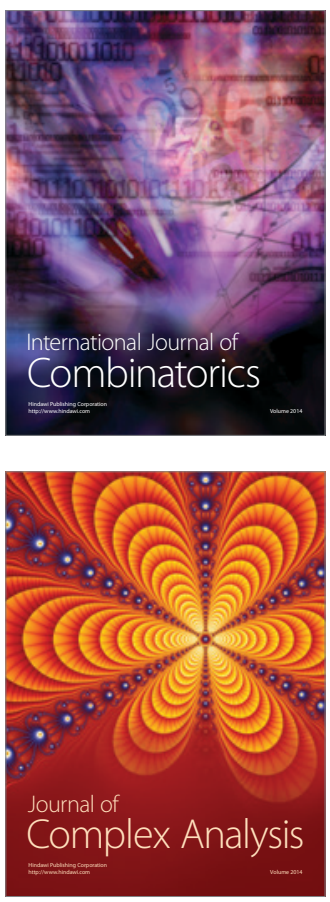

International Journal of

Mathematics and

Mathematical

Sciences
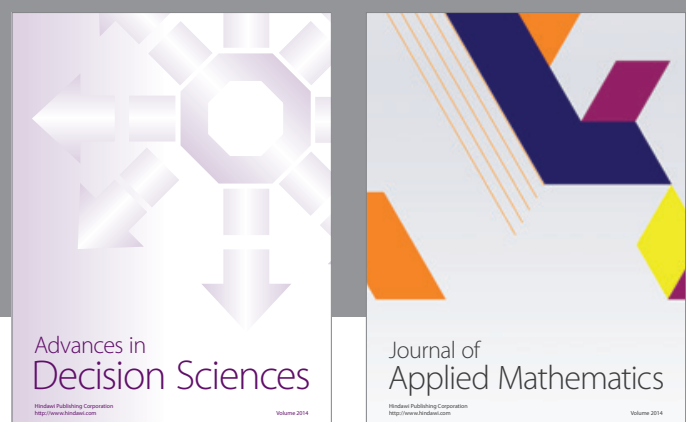

Journal of

Applied Mathematics
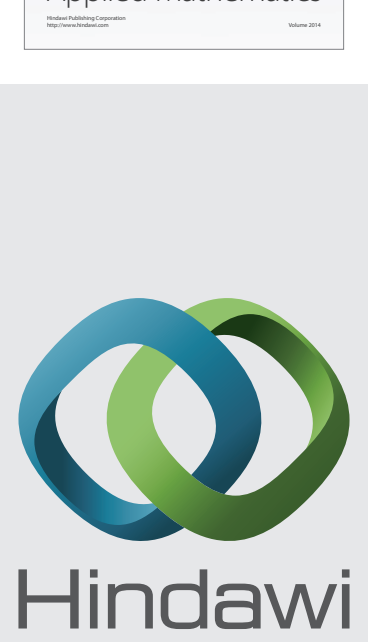

Submit your manuscripts at http://www.hindawi.com
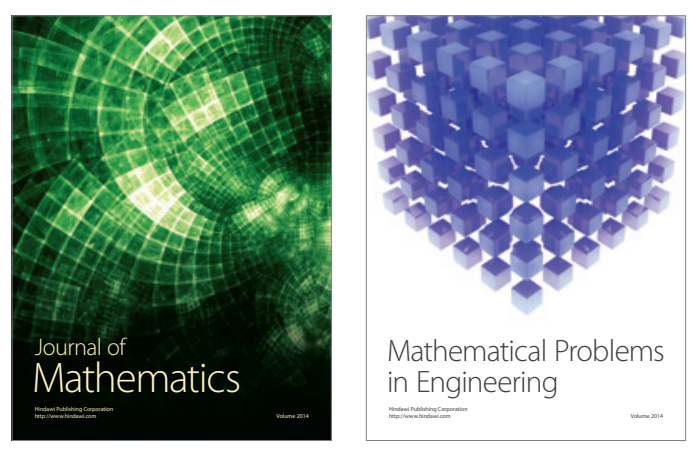

Mathematical Problems in Engineering
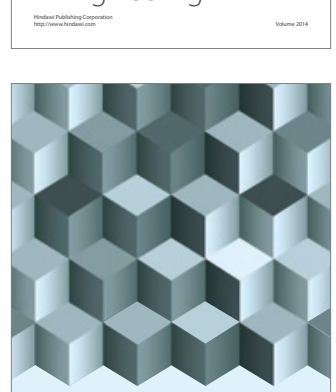

Journal of

Function Spaces
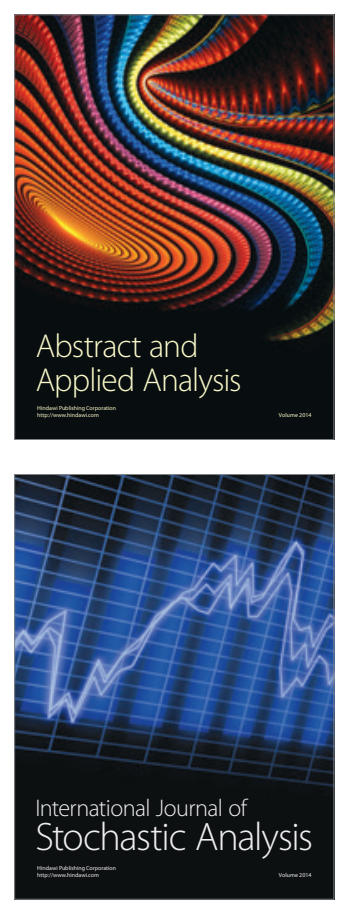

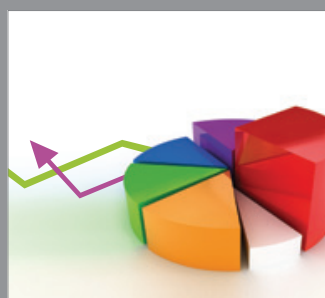

ournal of

Probability and Statistics

Promensencen
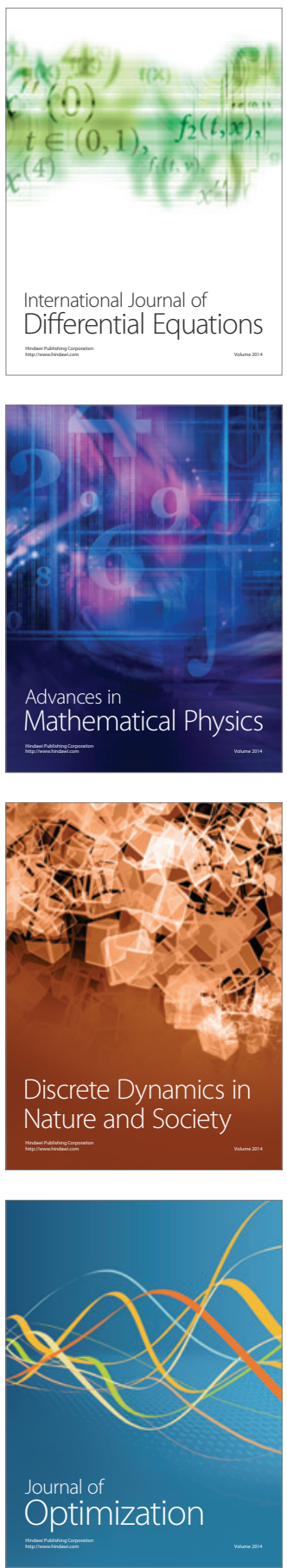